\title{
Unidirectional Vertical Emission from Photonic Crystal Nanolasers
}

\author{
Se-Heon Kim¹, Yong-Hee Lee ${ }^{2}$, Jingqing Huang', and Axel Scherer ${ }^{1}$ \\ ${ }^{1}$ Department of Electrical Engineering, California Institute of Technology, Pasadena, CA 91125, USA \\ Tel: (626) 395 2206,Fax: (626) 577 8442,e-mail: seheon@caltech.edu \\ ${ }^{2}$ Department of Physics, Korea Advanced Institute of Technology, Daejeon 305-701, South Korea
}

\begin{abstract}
Here, we emphasize the importance of using a bottom reflector to achieve unidirectional vertical emission from an ultra-small light emitter. Specifically, we have considered a photonic crystal slab nanocavity laser placed in the vicinity of a perfect mirror. By choosing near one-wavelength distance between the bottom reflector and the cavity, over $80 \%$ of photons generated inside the laser cavity can funnel into a small divergence angle of $\pm 30^{\circ}$. It is also interesting to observe that the natural radiation rate $(\sim 1 / Q$ factor $)$ of the nanocavity mode can be modified by varying the gap size, which is analogous to the famous cavity quantum electrodynamics effect for a point dipole source placed near a perfect mirror. A simple, comprehensive plane wave interference model is presented to explain the observed over six-fold vertical emission enhancement. Furthermore, we propose some of the very practical nanolaser designs based on a metal bonding technology, which may enable continuous current injection operation at room-temperature.
\end{abstract}

\section{INTRODUCTION}

Various high- $Q$ nanocavities that are based on either photonic bandgap effect or plasmonic confinement have been the subject of intense research topics in nanophotonics [1-3]. In such high- $Q$, small- $V$ cavities, spontaneous emission ( $\mathrm{SpE}$ ) rate of an excited atom is no longer the same as that of an atom in free space. Of particular importance is the enhancement of $\mathrm{SpE}$ into a specific cavity mode of interest, by which some of the ultimate photon sources with near unity SpE coupling factor $(\beta \sim 1)$ can be achieved [4]. Furthermore, such nanocavitycontrolled enhanced $\mathrm{SpE}$ rate may pave a new route towards $>100 \mathrm{~Gb} / \mathrm{s}$ ultra-high speed modulation of semiconductor lasers [5].

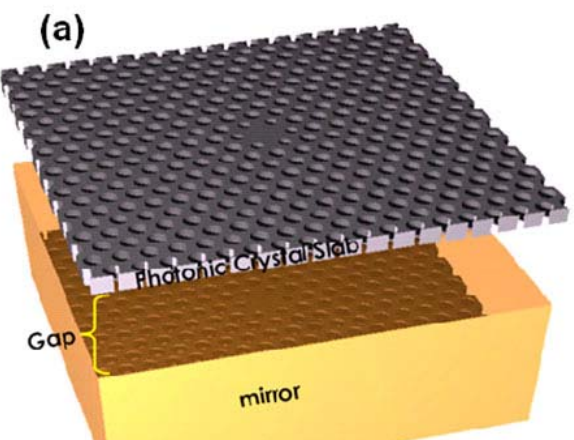

(b)
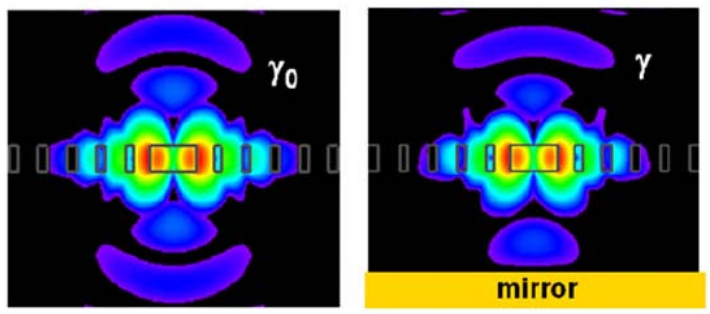

Figure 1: (a) A nanocavity formed in a thin photonic crystal membrane is suspended in air in the vicinity of a bottom reflector; (b) Electric-field intensity distributions $(|E| 2)$ of the deformed hexapole modes detected in the vertical cross sectional plane. Natural decay rate $\left(\gamma_{0}\right)$ of the nanocavity mode is now changed to $\gamma$ by the presence of the bottom reflector.

Photonic crystals (PhCs) have already proven their ability to efficiently confine photons in a sub-wavelength region. For example, the hexapole mode cavity can be designed to have a $Q$ factor of as high as $2 \times 10^{6}$ while its modal volume is kept to be as small as $\sim(\lambda / n)^{3}[6]$, which is well suited for various cavity quantum electrodynamics (cQED) experiments in both weak and strong coupling regimes. In fact, for the realization of efficient single photon sources based on $\mathrm{PhC}$ nanocavities, we do not need to rely on such high $Q$ factors; a moderate $Q$ factor of $\sim 1000$ is still large enough to demonstrate strong $\mathrm{SpE}$ enhancement due to their extremely small modal volume [7]. Moreover, the importance of a smaller mode volume over a higher $Q$ factor for some cQED experiments and nanolaser applications has been emphasized in recent studies [3, 8].

However, there still remain several critical issues that need to be addressed for real practical applications. 1) Nanocavity based photon sources have very poor directional emission, inherent to their sub-wavelength size. 2) Moreover, they usually have very poor thermal characteristics. A typical value for the thermal resistance of $\mathrm{PhC}$ slab nanolasers is $\sim 10^{6} \mathrm{~K} / \mathrm{W}$ [3]. 3) Finally, where and how to place electrical contacts for feasible current injection operation are still nontrivial issues. The original cavity $Q$ factor should not be sacrificed too much by the presence of the metal/dielectric contacts. Some partial answers for each of these three issues were provided 
by several authors $[1,3,9]$; however, we still lack an ultimate nanocavity design that solves all of these issues at once.

\section{A PHOTONIC CRYSTAL NANOCAVITY AS AN ARTIFICIAL ATOMIC EMITTER}

Most PhC nanocavities are formed in a thin dielectric slab suspended in air as depicted in Fig. 1a. Now let us assume the presence of a bottom reflector that is few $\mu \mathrm{m}$ away from the slab. Such a reflector is, in fact, included in most PhC slab structures, in the form of a bottom substrate. Since the refractive index of semiconductor used for $\mathrm{PhC}$ devices is rather high $(n>3)$, even a simple flat substrate may work as a good reflector [9, 10]. To increase the reflectivity, one may introduce alternating high/low refractive index materials during epitaxial growth processes to form a distributed Bragg reflector (DBR) [7,9]. Recently, we have already proposed and demonstrated the use of a bottom reflector to engineer originally diffused and highly divergent far-field emission into well-directed vertical beaming [9]. It has also been shown that over $80 \%$ of photons generated inside the nanocavity can be funnelled into a small divergence angle of $\pm 30^{\circ}$. Here, we would like to emphasize that the nanocavity mode can be treated as an artificial atom whose resonance linewidth is much sharper than that of the planar mirror (a condition for the weak-coupling regime of cQED), which is analogous to the well-known cQED example of a point dipole located near a perfect mirror [11]. Therefore, one can expect that similar modifications on radiation characteristics, such as radiation rates and emission profiles, would be observed from the $\mathrm{PhC}$ nanocavity mode.

(a)

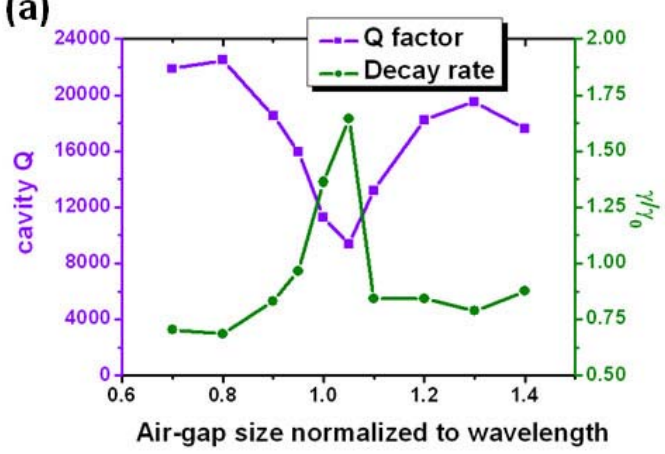

(b)
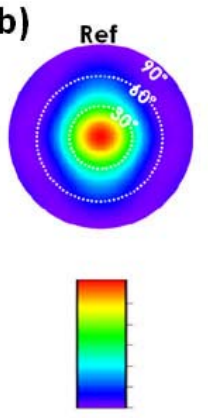
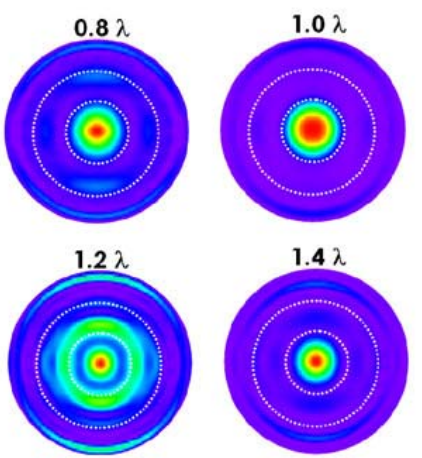

Figure 2: (a) Calculated cavity $Q$ factors and normalized decay rates $\left(\gamma / \gamma_{0}\right)$ of the deformed hexapole mode as a function of the air gap size; (b) Calculated far-field patterns for various values of the air-gap size. For comparison, the far-field pattern in the absence of the bottom reflector is shown together.

Figure 2a shows calculated $Q$ factors as we vary the air-gap size, assuming a perfect conductor as a bottom reflector. It should be noted that all numerical simulations presented in this paper were performed by using 3-D finite-difference time-domain method (FDTD). From the $Q$ factors, radiation rates of a $\mathrm{PhC}$ nanocavity mode $(\gamma)$ are obtained through the relation, $\gamma=\omega / Q$, where $\omega$ is the angular frequency of the nanocavity mode, and then normalized to the natural radiation rate of a $\mathrm{PhC}$ nanocavity mode $\left(\gamma_{0}\right)$ in the absence of the mirror. Figure $2 \mathrm{a}$ clearly shows that there is enhancement/suppression of radiation rate depending on the air-gap size. Moreover, from the calculated far-field radiation patterns (see Fig. 2b) [9], concentric ring-type modulations are clearly shown, indicating that there must be an interference effect due to the bottom reflector.

One important difference from the atomic dipole emission is that the $\mathrm{PhC}$ nanocavity mode is not a simple dipole emitter but a composite of electric and magnetic multipoles [9]. Thus, one may understand the observed phenomena in view of sum of interactions between each multipole component and the mirror. On the other hand, one may adopt a different view point that is based on the fact that the $\mathrm{PhC}$ resonant mode can be decomposed into plane waves, which will be the topic in the following section.

\section{PLANEWAVE INTERFERENCE MODEL}

The far-field simulation results in Fig. $2 b$ show that optimum vertical beaming effect can be obtained from $\sim \lambda$ air-gap size. To quantify the observed beaming results, we have calculated vertical emission enhancement factor, $W$, by dividing the calculated far-field vertical emission power, $P(\theta=0)$, by the corresponding reference power without the reflector, $P_{0}(\theta=0)$. It should be noted that the total energy stored in the resonant mode was kept to be almost constant (errors less than $2 \%$ ) as we varied the air-gap size. As shown in Fig. 3a (red line), when the air-gap is chosen to be resonant with the emission wavelength $\lambda, W$ is maximized to be $>6$. It is interesting to note that the optimum vertical enhancement is greater than 4 , the value that would result from a two-beam interference model [9]. Moreover, there exists a broad acceptable range of air-gap sizes, that would give $W>4$. Thus, experimental realization of such enhanced vertical beaming is rather robust against a possible deviation from the $1 \lambda$ air-gap [10]. 


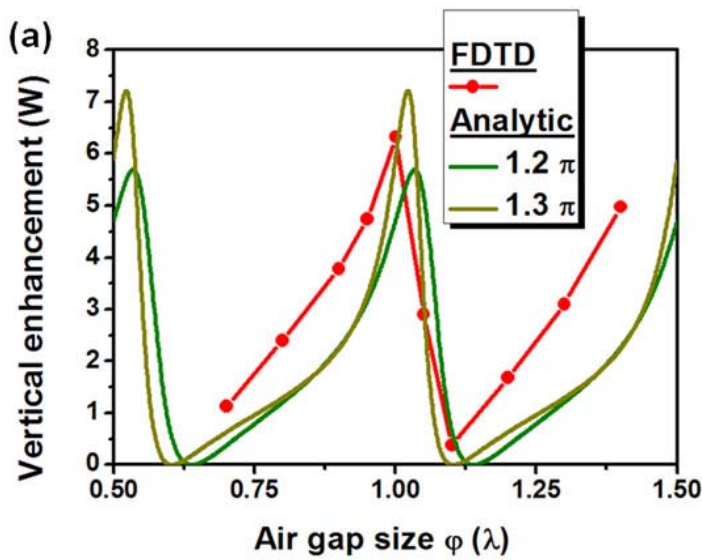

(b)

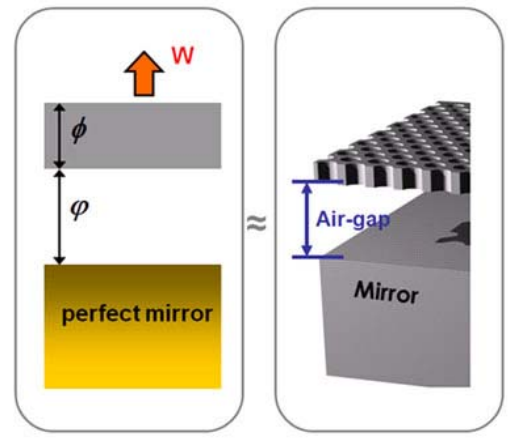

Figure 3: (a) Vertical emission enhancement factors (W) estimated from 3-D FDTD computations are compared with the results obtained by using a simple plane wave interference model; (b) A photonic crystal slab can be approximated as a dielectric slab with an effective refractive index. Then, $W$ is derived from the plane wave interference model, in which all possible multiple reflection/transmission processes in the slab and the air-gap are considered.

Now, let us approximate the $\mathrm{PhC}$ slab as a uniform dielectric slab having an effective refractive index and the $\mathrm{PhC}$ resonant mode as a point emitter embedded in the middle of the slab (see Fig. 3b). Here, we consider only vertical radiation ( $\mathrm{dc}$ component of plane waves, $\theta=0^{\circ}$ ). The downward propagating wave is redirected by the reflector underneath and interferes with the originally upward propagating wave. Considering an infinite sum of multiple reflection and transmission processes in the slab and in the air-gap, one obtains the following analytic expression for $W[9]$

$$
W=\left|1+\frac{t_{0}^{2} e^{i \phi}}{\left(1-r_{0}^{2} e^{2 i \phi}\right)\left(r_{0}-e^{-2 i \varphi}\right)-r_{0} t_{0}^{2} e^{2 i \phi}}\right|^{2},
$$

where $r_{0}$ and $t_{0}$ are coefficients of amplitude reflection and transmission for a single dielectric interface, respectively. $2 \varphi$ is a round-trip phase in the air-gap and $\phi$ is the phase change through the PhC slab. We plot the results for PhC slab thicknesses of $1.2 \pi$ and $1.3 \pi$ in Fig. 3a, which agree very well with the 3-D FDTD result. In fact, according to equation (1), the condition for enhanced vertical radiation is given by $2 \varphi=m \pi$, where $m$ is an integer. Thus, the use of a $\sim \lambda / 2$ air-gap size would also give similar enhanced beaming effect.

(a)

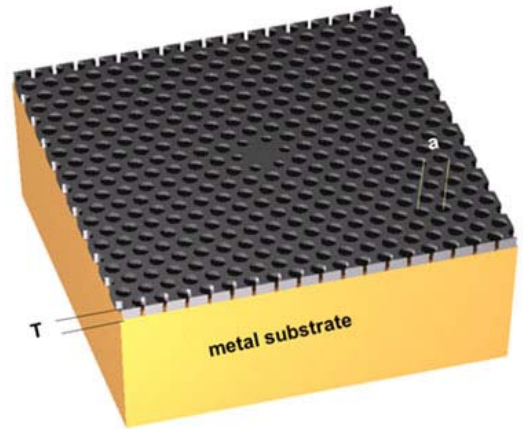

(b)

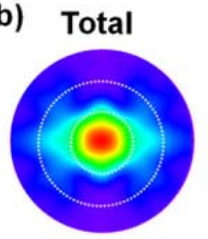

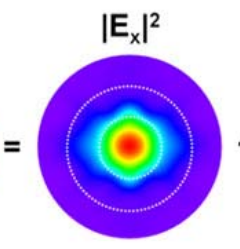

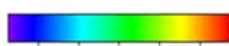

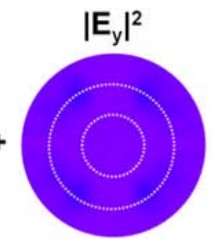

Figure 4: (a) Metal-bonded photonic crystal nanocavity may be considered as a special case with zero air-gap for the structure shown in Fig. 1a; (b) By optimizing the slab thickness, linearly-polarized, unidirectional farfield emission can be obtained, where the lattice constant and the slab thickness are assumed to be $380 \mathrm{~nm}$ and $350 \mathrm{~nm}$, respectively, with the resultant emission wavelength of $\sim 1430 \mathrm{~nm}$.

It is worth mentioning the recent experimental realization of unidirectional $\mathrm{PhC}$ nanolasers emitting at near $1.55 \mu \mathrm{m}$ based on InGaAsP/InP material systems [10]. The modified hexapole mode was excited as a laser mode [9] and a simple flat InP substrate was employed as a bottom reflector. The air-gap size was designed to be $\sim 860 \mathrm{~nm}$, which is roughly $\sim \lambda / 2$. Although the InP substrate used here has only $\sim 30 \%$ reflectivity and the airgap size is made $\sim 10 \%$ larger than the optimum thickness, fairly good directional far-field pattern has been obtained, in which about $56 \%$ photons can be collected within a small divergence angle of $\pm 30^{\circ}$. 


\section{PROSCPECTS}

According to the simple plane wave interference model, the condition for air-gap size that gives well-directed vertical beaming is given as $2 \varphi=m \pi$. Then, what would happen if we had zero air-gap which corresponds to the $m=0$ case? As shown in our previous results [9], when the bottom reflector is made of dielectric materials (e.g., simple dielectric substrate or DBR), cavity $Q$ tends to decrease to zero as the air-gap size approaches to zero. However, one may find out some resonant modes that have large enough $Q$ factors for actual lasing if the bottom reflector could be replaced by silver or gold, which is almost a perfect reflector near infrared wavelengths.

Figure 4a shows a schematic for such a metal-bonded PhC nanocavity. This metal layer can provide highly efficient conduction pathways both for current and heat flows while it does not degrade the $Q$ factor too much. Furthermore, the flat metal surface can be used to enhance vertically emitted output power, enabling fairly good unidirectional vertical emission as presented in Fig. 4b. $Q$ factors in the range of $500 \sim 700$ are usually achievable by using gold. Further increase of the $Q$ factor is expected by employing a thin-oxide layer positioned between the gold mirror and the PhC slab. We are currently working towards the realization of practical current injection nanolasers based on this metal-bonded structure and further details will be reported in our forthcoming papers.

Finally, it should be note that the proposed vertical beaming scheme is not limited to this particular nanocavity geometry but may be applied to more general classes of nano- and micro-cavities, including plasmonic nanocavities, plasmon antennas, microdisks, and microspheres.

\section{ACKNOWLEDGEMENTS}

This work was supported in part by the Air Force Office of Scientific Research (FA9550-04-1-0434), the Army Research Office (W911NF-07-1-0277), and the National Science Foundation (EEC-0812072). We gratefully acknowledge critical support and infrastructure provided for this work by the Kavli Nanoscience Institute at Caltech.

\section{REFERENCES}

[1] H.-G. Park et al.: Electrically driven single-cell photonic crystal laser, Science, vol. 305. pp. 1444-1447, Sept. 2004.

[2] M. Hill et al.: Lasing in metallic-coated nanocavities, Nature Photon., vol. 1. pp. 589-594, Sept. 2007.

[3] K. Nozaki et al:: Room temperature continuous wave operation and controlled spontaneous emission in ultrasmall photonic crystal nanolaser, Opt. Express, vol. 15. pp. 7506-7514, June 2007.

[4] H. Yokoyama : Physics and device applications of optical microcavities, Science, vol. 256. pp. 66-70, 1992.

[5] H. Altug et al:: Ultrafast photonic crystal nanocavity laser, Nat. Physics, vol. 2. pp. 484-488, July 2006.

[6] H.-Y. Ryu et al.: High-quality-factor and small-mode-volume hexapole modes in photonic-crystal-slab nanocavities, Appl. Phys. Lett. , vol. 83. pp. 4294-4296, Nov. 2003.

[7] S.-H. Kim et al.: Toward efficient unidirectional photonic crystal light emitters, Proc. SPIE, vol. 6352. 635213, 2006.

[8] H.-Y. Ryu et al:: Enhancement of spontaneous emission from the resonant modes of a photonic crystal slab single-defect cavity, Opt. Lett. , vol. 28. pp. 2390-2392, Dec. 2003.

[9] S.-H. Kim et al.: Vertical beaming of wavelength-scale photonic crystal resonators, Phys. Rev. B, vol. 73. 235117, June 2006.

[10] J.-H. Kang et al:: Polarized vertical beaming of an engineered hexapole mode laser, Opt. Express, vol. 17. pp. 6074-6081, Mar. 2009.

[11] E. A. Hinds: in Cavity Quantum Electrodynamics, edited by P. R. Berman (Academic Press, New York, 1994). 